\title{
RESPONSES TO ANTI-ADBLOCK FILTERS: THEORETICAL FOUNDATIONS, INFLUENTIAL FACTORS, AND MANAGERIAL IMPLICATIONS
}

\author{
Ignacio REDONDO (D) ${ }^{*}$, Gloria AZNAR (D)2 \\ ${ }^{1}$ Departamento de Financiación e Investigación Comercial, \\ Universidad Autónoma de Madrid, Madrid, Spain \\ ${ }^{2}$ Departamento de Marketing e Investigación Comercial, \\ Universidad CEU San Pablo, Madrid, Spain
}

Received 10 March 2020; accepted 09 September 2020

\begin{abstract}
Ad-supported websites face an increasing loss of monetizable ad impressions due to the rapid spread of adblockers, which allow users to get desired website content without unwanted advertising. As a countermeasure, many of these websites use anti-adblock filters, which detect adblock users and prevent their access to website content unless their adblockers are disabled. Users may certainly respond by disabling their adblockers but also by leaving the website or trying to bypass the anti-adblock filter. To better understand the choice among these responses, we propose a conceptual framework that combines psychological reactance theory along with uses and gratifications theory. We also hypothesize the influence of four user-related factors: (a) more positive (negative) attitudes toward online advertising encourage adblocker deactivation (website abandonment); (b) longer adblock usage experiences enable filter bypassing; (c) wider (narrower) scopes of online activities stimulate filter bypassing (website abandonment); and (d) greater online privacy concerns discourage adblocker deactivation. These hypotheses were supported by a survey conducted by the Spanish advertising industry, but the influence of breadth of online activities was negligible in practice. Our findings suggest the importance of improving attitudes toward online advertising, reducing online privacy concerns, and searching for alternative ways to monetize website visits.
\end{abstract}

Keywords: anti-adblocking, advertising avoidance, online privacy, advertising management, psychological reactance, uses and gratifications theory.

JEL Classification: D91, L86, M37.

Online supplementary material: Supporting information for this paper is available as online supplementary material at https://doi.org/10.3846/jbem.2020.13698

${ }^{\star}$ Corresponding author. E-mail: ignacio.redondo@uam.es

Copyright (c) 2020 The Author(s). Published by Vilnius Gediminas Technical University

This is an Open Access article distributed under the terms of the Creative Commons Attribution License (http://creativecommons. org/licenses/by/4.0/), which permits unrestricted use, distribution, and reproduction in any medium, provided the original author and source are credited. 


\section{Introduction}

Adblockers are rapidly becoming popular as freely available and easy-to-use software tools that allow Internet users (henceforth "netizens") to avoid unwanted ad impressions during their browsing sessions, with a degree of effectiveness that no mechanical means has previously allowed in the realm of offline media (e.g., remote controls and video recorders with respect to television commercials) (Redondo \& Aznar, 2018). The growing popularity of adblockers is also motivated by their abilities (a) to filter the advertising formats that most bother/interrupt netizen browsing experiences, (b) to reduce the number of third-party tracking cookies that threaten netizen privacy, and (c) to increase website loading speed and reduce waste of resources such as bandwidth and computing capacity (Zhu et al., 2019).

The spread of adblockers is leading to increased losses of monetizable ad impressions, which hurt the main income source of ad-supported website publishers and may subsequently undermine investments in content quality designed to continue attracting netizens (Shiller et al., 2018). In order to reduce the loss of ad impressions, many ad-supported websites are using anti-adblock filters to detect the presence of adblockers and prevent their users from accessing the website content unless such adblockers are disabled. But these filters are double-edged swords that, depending on the responses by adblock users, can cause both an increase in ad impressions and a reduction in website visitors. Adblock users can basically choose from among three possible responses: disabling their adblockers, leaving the website, or trying to bypass the anti-adblock filter, either by installing an anti-adblock killer script, or by using manual methods such as turning off JavaScript, deleting the anti-adblock warning from the page's elements, and refreshing the page and quickly enabling the adblocker before ads appear on the webpage. From the standpoint of website managers, disabling the adblocker is the optimal response because it allows users to visit the website and generate monetizable ad impressions; leaving the website is the worst response because it means losing both the ad impressions and the website visitors; and bypassing the anti-adblock filter is a lukewarm response because it doesn't generate ad impressions but keeps the visitors, who thus are part of the website audience and are reachable through alternative advertising formats.

Recent studies have called for research on how to mitigate the imbalance that adblocking and anti-adblocking technologies have caused in the online advertising market (Aseri et al., 2020; Gordon et al., 2020). Within this vast and unexplored field of research, the present study focuses on the conflict that arises when adblock users try to access websites with anti-adblock filters, and it has a triple objective: (a) to deepen the theoretical understanding of the motives of netizens behind their responses to anti-adblock filters, (b) to identify some attitudinal and behavioral factors that drive netizens' responses to anti-adblock filters, and (c) to discuss the management implications for online advertising stakeholders. With a similar motivation, Söllner and Dost (2019) found remarkable evidence that (a) willingness to disable the adblocker is stimulated by the intention of supporting the website publisher and the expectation of receiving more useful, less disruptive, and more discreet ads, and (b) the rate of adblocker deactivation increases when users receive appropriate anti-adblock message appeals from the website. In turn, the present study makes four original contributions to the current knowledge:

- development of a conceptual framework in which netizen motivations and reactions to anti-adblock filters are explained by the combination of psychological reactance theory and uses and gratifications theory; 
- analysis of the three possible responses to anti-adblock filters and finding that the attempt to bypass the filter, despite being the least frequent and least known response, is already important for management and will likely be more important due to its foreseeable growth in the coming years;

- disclosure of the significant role played by two attitudinal factors: attitude toward online advertising, which is crucial in explaining the divergence between the decisions of adblocker deactivation and website abandonment, and online privacy concern, which is a moderate deterrent to adblocker deactivation;

- uncovering of the role played by one behavioral factor: adblock usage experience is a significant positive driver behind attempts to bypass the anti-adblock filter, which allows for predicting that this response will become more frequent as users accumulate more experience over time.

The rest of the paper follows a common structure. The next section provides theoretical background for understanding the responses to anti-adblock filters and hypothesizing the role of user-related factors. The Method section gives a detailed description of the data collection and analysis procedures for the assessment. The Results section presents the outcomes of the statistical analyses. The Discussion section provides interpretations of the findings and their managerial implications. The final section presents conclusions, limitations, and suggestions for future research.

\section{Conceptual framework and hypothesis development}

\subsection{Overview}

According to psychological reactance theory (Brehm \& Brehm, 1981), when people perceive that their freedom to choose or act is being threatened, they tend to undergo a motivational reaction ("reactance") that is intended to reaffirm their affected freedom. In the online environment, where users are usually very involved in achieving their hedonic/utilitarian goals with a high sense of freedom and control (Novak et al., 2000), forced exposure to unwanted advertising is often perceived as a hindrance to the tasks undertaken to achieve personal goals, all of which produces significant levels of psychological reactance (Edwards et al., 2002). Indeed, psychological reactance helps understand netizen avoidance of various types of interruptive/distracting advertising such as online behavioral (Ham, 2017) and Facebook newsfeed (Youn \& Kim, 2019) ads. Psychological reactance also helps understand why netizens become motivationally aroused to install and use adblockers in order to reestablish their perceived loss of freedom, maintain their effective control over unwanted advertising, and ultimately stay tightly focused on their online tasks (Redondo \& Aznar, 2018; Tudoran, 2019). Similarly, adblock users may experience psychological reactance whenever a website's anti-adblock filter threatens their control over unwanted advertising by imposing the deactivation of their adblockers as a precondition for accessing the website's content. Faced with this threat, adblock users may become motivationally aroused to choose the response that, as far as possible, best contributes to restoring their control over wanted content and unwanted ads.

Uses and gratifications theory assumes that audience members actively choose media that meet their self-defined goals in order to satisfy human needs such as information, en- 
tertainment, companionship, and escape (Rubin, 1983). Central to this theory is the notion that media choice is strongly influenced by the match or mismatch between gratifications sought (expected to be had with the media exposure) and gratifications obtained (actually experienced through the media exposure) (Palmgreen et al., 1985). Moreover, central to this study is the distinction between content gratifications, which refer to the intrinsic values that users find in the mediated messages themselves, and process gratifications, which refer to the satisfactions that users experience from being involved in the communication process itself (Stafford \& Stafford, 1996).

Uses and gratifications theory has been widely used to explain what users do with various traditional offline media, such as television (Rubin, 1983) and magazines (Payne et al., 1988). But this theory's applications to online media have been much more extensive because cyberspace allows for obtaining a larger variety of gratifications and experiencing higher levels of satisfaction (Ji \& Fu, 2013), and because online users show higher levels of interactivity and intentionality in their media behavior (Ruggiero, 2000). Indeed, this theory has helped to explain the practice of a wide range of online activities such as social networking (Smock et al., 2011) and e-shopping (Lim \& Ting, 2012).

In the realm of advertising avoidance, uses and gratifications theory has been used to explain why TV viewers use remote control devices to scan effortlessly other channels' programming during commercial blocks ("zapping") and to reduce advertising exposure time by fast-forwarding through commercial blocks when replaying their recorded programming (“zipping”) (Stafford \& Stafford, 1996). To date, however, online advertising avoidance through adblockers has not been grounded in this theory, which is an essential part of the conceptual framework proposed here to better understand netizen decisions about adblocking and anti-adblocking. On the basis of this theory, it can be argued that adblock users obtain content gratifications when they enjoy the content chosen during their web browsing and obtain process gratifications when they manage to enjoy such content without being interrupted/bothered by the ads filtered by their adblockers. Whenever adblock users try to access a website with anti-adblock filter, they feel that this filter is threatening their ability to obtain the desired content and/or process gratifications. If users disable their adblockers, they will obtain the content gratification (for the enjoyment of the content sought) but not the process gratification (for the enjoyment of such content without advertising), so that there will be only a partial match between their desired and actual results. If users try to bypass the anti-adblock filter, they will try to obtain both the content and process gratifications, which would produce a complete match between their desired and obtained gratifications. If users leave the website, they will obtain neither the content nor the process gratification, which will result in a complete mismatch between what they want and what they get. Within this conceptual framework, the following subsections discuss how four attitudinal/behavioral factors may influence the responses to anti-adblock filters.

\subsection{The role of attitude toward online advertising}

That many netizens have installed and are actually using adblockers manifests their deliberate resolution to systematically bypass the ads accompanying the content of websites visited. These users tend to experience psychological reactance whenever their ability to enjoy 
content without advertising is restricted by an anti-adblock filter. Among the three possible responses to an anti-adblock filter, there are two that indicate extreme reactions to online advertising. On the one hand, disabling the adblocker implies "opening the door" to all ads managed from the website visited as a counterpart to the enjoyment of the content provided there. On the other hand, leaving the website implies the radical decision to "close the door" to all ads managed from there, although this decision entails not accessing the content sought. The decision on one of these extreme responses is hypothetically conditioned by attitude toward online advertising, which, consistent with MacKenzie and Lutz (1989), is defined as a learned predisposition to respond in a consistently favorable or unfavorable manner toward Internet ads. Indeed, prior research consistently shows that responses of approach or avoidance to advertising are conditioned by attitude toward advertising, regardless of whether this attitude refers to advertising in general (Rojas-Méndez \& Davies, 2005) or advertising in specific media (Speck \& Elliott, 1997). Understandably, users favorably (unfavorably) predisposed to online advertising are more likely to disable their adblockers (leave the website) by more easily accepting (rejecting) the website's advertising exposure as a counterpart to the website's content enjoyment.

H1: Adblock users with more positive (negative) attitudes toward online advertising, in response to an anti-adblock filter, are more likely to disable their adblockers (leave the website)

\subsection{The role of adblock usage experience}

Getting started with adblockers is a relatively simple task that requires hardly any learning time (Redondo \& Aznar, 2018). However, bypassing anti-adblock filters is a much more complicated task that usually requires a considerable amount of experience. To complete this task successfully, novice users need learning time to acquire advanced knowledge through mass/interpersonal channels and to develop specific skills through sufficient training. They will also need an additional learning period whenever they face a new anti-adblock system with a more sophisticated filtering capacity. The longer netizens have been using adblockers, the more likely they would have accumulated the knowledge and skills required to effectively bypass anti-adblock filters. The successes achieved in performing this task will make users increase their perceived self-efficacy, which will be a strong psychological motivation to continue performing the same task in the future (Bandura, 1978). The same rationale has been used to explain that engaging in complex computer/Internet activities is proximately motivated by perceived computer/Internet self-efficacy and remotely influenced by computer/Internet use experience (Compeau et al., 1999; Eastin \& LaRose, 2000). Based on this reasoning, it is suggested that the longer the adblock usage experience is, the more likely the attempt to bypass anti-adblock filters will be.

$\mathrm{H} 2$ : When confronted with an anti-adblock filter, netizens who have been using adblockers for a longer period of time are more likely to engage in bypassing such a filter

\subsection{The role of breadth of online activities}

Individuals who perform a wide range of online activities manifest that they seek and obtain a great variety of gratifications in such activities, a variety that reasonably includes both con- 
tent and process gratifications. So these individuals are accustomed to successfully adjusting their sought gratifications with those they obtain in a wide variety of online activities. In addition, these individuals are characterized by high levels of perceived and actual internet skills (Hargittai \& Hinnant, 2008). It is then reasonable to expect that, whenever they face new challenges in navigation, they will be prone to using their digital skills to expand the variety of rewarding experiences. Thus, it may be assumed that those who perform a greater breadth of online activities will be more inclined to bypass the anti-adblock filter with the intention of obtaining both content gratification (access to content) and process gratification (control of advertising).

In the opposite case, users that perform less diverse activities on the Internet manage to satisfy a smaller variety of content and process gratifications. These users tend to engage in a smaller variety of online activities due to their lower levels of both digital literacy and self-efficacy in solving Internet-related problems (Helsper \& Eynon, 2013). Understandably, these users are accustomed to desisting from performing certain online activities in which they are unable to achieve a match between their sought and their obtained gratifications. Thus, it may be expected that these users will tend to be less averse to the simplest response, i.e., leaving the website, even if this decision results in a complete mismatch with the content and process gratifications they seek.

H3: Adblock users that perform a greater (smaller) breadth of online activities, in response to an anti-adblock filter, are more likely to engage in bypassing such a filter (leave the website)

\subsection{The role of online privacy concern}

Among other information-gathering technologies, advertising companies use third-party cookies to track netizens across multiple websites on which they serve ads, so that such companies can identify netizen needs and preferences in order to target and personalize advertising more accurately. Third-party cookies can constitute an invasion of privacy because they allow for collecting, transmitting, and sharing personal information without netizen knowledge or consent (Miyazaki, 2008). Netizens tend to develop privacy concerns when they perceive that (a) the collection and use of their personal information by third parties may have potentially negative consequences and (b) the ability to control how or when their personal information is disclosed or used, is virtually lost (Dinev \& Hart, 2004). Perceived loss of control over personal information collected by third-party advertising companies leads to an experience of psychological reactance, which motivates attempts to prevent or at least reduce privacy intrusion by advertisers (Bleier \& Eisenbeiss, 2015; Tucker, 2014). Netizens who are more concerned with online privacy tend to take more precautions to protect their personal information from third-party intrusion and tend to be more motivated to avoid online advertising (Boerman et al., 2017; Tudoran, 2019). Following this line of reasoning, it may be assumed that adblock users with more online privacy concern will be less inclined to disable their adblockers because this response would expose them to the website's ads, which could be used to track their browsing behavior.

H4: Adblock users who are more concerned about online privacy, in response to an antiadblock filter, are less likely to disable their adblockers 


\section{Method}

\subsection{Data}

Data came from the $20^{\text {th }}$ edition of Navegantes en la Red (Surfers on the Web), a survey conducted annually in Spain by AIMC (Asociación para la Investigación de Medios de Comunicación). AIMC is a non-profit and non-affiliated organization that represents the Spanish advertising industry's stakeholders. As a joint industry committee, AIMC is entrusted with the planning, execution, control, and publication of media audience measurements that are then used to plan advertising campaigns. AIMC's members contribute to the financing of its media audience surveys and are democratically represented in its decision-making structures, all of which endorse the transparency of its research procedures.

Navegantes en la Red is a self-administered online survey in which a large sample of Spanish website users are asked about their attitudes, beliefs, and behaviors about the Internet. Participants are recruited through four means: (a) banners/links/news located on more than two hundred popular Spanish websites and on some of their profiles in Facebook, Twitter, etc.; (b) emails sent by AIMC to the participants in this survey's latest editions who expressed their willingness to continue participating in the future; (c) invitations to the members of the online panel that Research Now SSI manages in Spain; and (d) screen messages displayed to the active users of a popular Spanish-language chat website. To encourage participation, several state-of-the-art smartphones are raffled among those individuals who adequately complete the questionnaire.

\subsection{Participants}

During the period the survey's website was accessible (17 October - 10 December 2017), a total of 15,896 questionnaires were collected, but 644 had to be excluded due to substantial missing data or inconsistent responses. The final sample thus consisted of 15,252 valid questionnaires, $47 \%$ of which came from visitors to the Spanish websites, $29 \%$ from previous participants in the survey, $19 \%$ from members of the online panel, and $5 \%$ from users of the chat website. In terms of gender, $67 \%$ of the participants were male and $33 \%$ female. By age, $13 \%$ of the participants were under 25 years old, $21 \%$ were between 25 and 34, 28\% were between 35 and 44,23\% were between 45 and 54, and 15\% were over 54 years old.

\subsection{Variables}

Participants who reported using adblockers in a filter question were then asked for (a) their most frequent response when coming across websites with anti-adblock filters and (b) how long they have been using adblockers. Response to anti-adblock filters was measured on a nominal scale with three categories $(1=$ disable the adblocker, $2=$ try to bypass the antiadblock filter, and 3 = leave the website). Adblock usage experience was defined as the number of years using adblockers and reported on a five-point ordinal scale ( $1=$ less than 1 year, $2=$ between 1 and 2 years, 3 = between 2 and 3 years, $4=$ between 3 and 5 years, and $5=$ more than 5 years). 
Two relatively abstract variables were measured through multiple items gathered from the survey (Appendix). Firstly, consistent with Wang and Sun (2010), Attitude toward online advertising was calculated by averaging the responses to four items rated on a four-point ordinal scale $(1=$ not agree at all, $2=$ slightly agree, $3=$ fairly agree, and $4=$ strongly agree $)$. Secondly, consistent with Bleier and Eisenbeiss (2015), Online privacy concern was computed by averaging the responses to four items scored on a five-point Likert scale (from $1=$ strongly disagree, to 5 = strongly agree).

Moreover, consistent with Redondo and Charron (2013), Breadth of online activities was calculated by counting how many of the 34 activities listed in the questionnaire (Appendix) had been performed by the respondent on the Internet in the last 30 days.

\subsection{Statistical analyses}

To assess the reliability of multi-item measures, Cronbach's alpha coefficients were calculated and the recommended value of 0.7 (Hair et al., 2010) was used as a threshold above which the combined items have sufficient internal consistency.

As a preliminary step to hypothesis testing, some descriptive statistics were computed for the three response groups (disable the adblocker, try to bypass the anti-adblock filter, and leave the website) across the four proposed predictors (Attitude toward online advertising, Adblock usage experience, Breadth of online activities, and Online privacy concern).

Further, to simultaneously test the hypotheses and assess the magnitude of all relationships, an unordered multinomial logistic regression model was built in which the unordered three-category dependent variable (response groups) was explained by the four numerical independent variables (predictors). Multinomial logistic regression uses the maximum likelihood estimation method, which maximizes the probability of getting the observed results given the fitted regression coefficients. Multinomial logistic regression measures model estimation fit with the value of -2 times the log of the likelihood value $(-2 L L)$. The lower the $-2 L L$ value is, the better the model fit is (the minimum value for $-2 L L$ is zero, which corresponds to a perfect fit). So, the contribution of each predictor to the model fit improvement was evaluated through the change in $-2 L L$ resulting from adding such a predictor, a change that was tested by the chi-square test.

As multinomial logistic regression is an extension of binary logistic regression, the three categories of the dependent variable could be compared through three binary logistic regressions (disable the adblocker vs. try to bypass the anti-adblock filter, disable the adblocker vs. leave the website, and try to bypass the anti-adblock filter vs. leave the website). The coefficients of each binary logistic regression are straightforward, robust, and easy to interpret: (a) the sign of the $B$ coefficient reflects the direction of the relationship, so that a positive (negative) coefficient indicates that the first group scores higher (lower) on the predictor than the second group; (b) the Wald statistic tests the significance of the $B$ coefficient, so that a positive test indicates that the predictor contributes to explaining group differences more than would be expected by chance; and (c) the value of the $\operatorname{Exp}(B)$ coefficient reflects the magnitude (in terms of odds) of the relationship, so that $(\operatorname{Exp}(B)-1) \times 100$ equals the percentage change in odds between groups for each unit change in the predictor. Another very convenient feature of the logistic regression is its 
lack of strict assumptions, such as multivariate normality and equal variance-covariance matrices across groups (Hair et al., 2010).

All statistical analyses were performed using SPSS for Windows (version 22, IBM SPSS, Armonk, NY, USA, 2013). Significance level was set at $p<.05$.

\section{Results}

A total of 8,230 participants reported being adblock users. In relation to their most frequent response to an anti-adblock filter, 3,573 reported disabling the adblocker, 1,247 trying to bypass the anti-adblock filter, and 3,236 leaving the website, while 173 reported having never found any anti-adblock filter and 1 gave no answer. These last 174 participants were excluded from the statistical analyses presented below.

With respect to the reliability of multi-item scales, Cronbach's alpha equaled 0.75 for Attitude toward online advertising and 0.73 for Online privacy concern, both above the threshold value, suggesting acceptable levels of internal consistency.

Table 1. Descriptive statistics for responses to anti-adblock filters across predictors

\begin{tabular}{|c|c|c|c|}
\hline & $\begin{array}{l}\text { Disable the } \\
\text { adblocker }\end{array}$ & $\begin{array}{l}\text { Try to bypass the } \\
\text { anti-adblock filter }\end{array}$ & Leave the website \\
\hline \multicolumn{4}{|c|}{ Attitude toward online advertising } \\
\hline Mean & 2.46 & 2.25 & 2.17 \\
\hline Standard deviation & 0.61 & 0.61 & 0.65 \\
\hline Sample size & 3,536 & 1,236 & 3,205 \\
\hline \multicolumn{4}{|c|}{ Adblock usage experience } \\
\hline Mean & 2.98 & 3.40 & 3.24 \\
\hline Standard deviation & 1.40 & 1.37 & 1.41 \\
\hline Sample size & 3,560 & 1,242 & 3,221 \\
\hline \multicolumn{4}{|c|}{ Breadth of online activities } \\
\hline Mean & 12.16 & 12.68 & 11.70 \\
\hline Standard deviation & 5.00 & 5.32 & 5.01 \\
\hline Sample size & 3,573 & 1,247 & 3,236 \\
\hline \multicolumn{4}{|l|}{ Online privacy concern } \\
\hline Mean & 3.99 & 4.16 & 4.15 \\
\hline Standard deviation & 0.83 & 0.75 & 0.83 \\
\hline Sample size & 3,572 & 1,246 & 3,234 \\
\hline
\end{tabular}

Table 1 presents the mean differences in responses to anti-adblock filters based on the available sample with no-missing values for the corresponding predictor. Attitude toward online advertising showed the highest mean among those disabling the adblocker ("adblocker disablers") and the lowest among those leaving the website ("website leavers"). Adblock usage experience had the highest mean among those trying to bypass the ad-block filter ("filter bypassers") and the lowest among adblocker disablers. Breadth of 
online activities displayed the highest mean for filter bypassers and the lowest for website leavers. Online privacy concern had the lowest mean for adblocker disablers and similar mean values for the other groups.

Table 2 presents the results of the multinomial logistic regression model estimated over the available sample with no-missing values for all predictors. Likelihood ratio tests indicate that each predictor made a significant individual contribution to explaining the whole set of between-group differences, but the contribution sizes varied substantially: while Attitude toward online advertising stood out with the highest chi-square value and Adblock usage experience showed an intermediate value, Breadth of online activities and Online privacy concern had much lower values.

Table 2. Multinomial logistic regression for responses to anti-adblock filters $(\mathrm{N}=7,943)$

\begin{tabular}{|c|c|c|c|c|c|c|}
\hline \multicolumn{7}{|c|}{ Likelihood ratio tests } \\
\hline Effect & \multicolumn{2}{|c|}{$\begin{array}{c}-2 L L \text { of reduced } \\
\text { model }\end{array}$} & \multicolumn{2}{|c|}{ Chi-square } & $d f$ & Sig. \\
\hline Intercept & \multicolumn{2}{|c|}{$12,646.43$} & \multicolumn{2}{|c|}{69.04} & 2 & $p<0.01$ \\
\hline Attitude toward online advertising & \multicolumn{2}{|c|}{$12,883.54$} & \multicolumn{2}{|c|}{306.15} & 2 & $p<0.01$ \\
\hline Adblock usage experience & \multicolumn{2}{|c|}{$12,654.65$} & \multicolumn{2}{|c|}{77.26} & 2 & $p<0.01$ \\
\hline Breadth of online activities & \multicolumn{2}{|c|}{$12,601.43$} & \multicolumn{2}{|c|}{24.04} & 2 & $p<0.01$ \\
\hline Online privacy concern & \multicolumn{2}{|c|}{$12,607.93$} & \multicolumn{2}{|c|}{30.55} & 2 & $p<0.01$ \\
\hline \multicolumn{7}{|c|}{ Parameter estimates } \\
\hline Adblocker disablers vs. Filter bypassers & $B$ & Std. Error & Wald & $d f$ & Sig. & $\operatorname{Exp}(B)$ \\
\hline Intercept & 1.35 & 0.25 & 29.91 & 1 & $p<0.01$ & \\
\hline Attitude toward online advertising & 0.52 & 0.06 & 87.93 & 1 & $p<0.01$ & 1.67 \\
\hline Adblock usage experience & -0.19 & 0.03 & 56.88 & 1 & $p<0.01$ & 0.83 \\
\hline Breadth of online activities & -0.02 & 0.01 & 5.99 & 1 & $p<0.05$ & 0.98 \\
\hline Online privacy concern & -0.18 & 0.04 & 16.76 & 1 & $p<0.01$ & 0.84 \\
\hline Filter bypassers vs. Website leavers & $B$ & Std. Error & Wald & $d f$ & Sig. & $\operatorname{Exp}(B)$ \\
\hline Intercept & -2.04 & 0.25 & 66.26 & 1 & $p<0.01$ & \\
\hline Attitude toward online advertising & 0.18 & 0.06 & 10.88 & 1 & $p<0.01$ & 1.20 \\
\hline Adblock usage experience & 0.06 & 0.03 & 5.73 & 1 & $p<0.05$ & 1.06 \\
\hline Breadth of online activities & 0.03 & 0.01 & 22.35 & 1 & $p<0.01$ & 1.03 \\
\hline Online privacy concern & 0.02 & 0.04 & 0.26 & 1 & 0.61 & 1.02 \\
\hline Adblocker disablers vs. Website leavers & $B$ & Std. Error & Wald & $d f$ & Sig. & $\operatorname{Exp}(B)$ \\
\hline Intercept & -0.68 & 0.18 & 14.36 & 1 & $p<0.01$ & \\
\hline Attitude toward online advertising & 0.70 & 0.04 & 279.77 & 1 & $p<0.01$ & 2.01 \\
\hline Adblock usage experience & -0.13 & 0.02 & 47.60 & 1 & $p<0.01$ & 0.88 \\
\hline Breadth of online activities & 0.02 & 0.01 & 9.30 & 1 & $p<0.01$ & 1.02 \\
\hline Online privacy concern & -0.15 & 0.03 & 23.73 & 1 & $p<0.01$ & 0.86 \\
\hline
\end{tabular}


Parameter estimates indicate the predictor influence on each binary logistic regression. Regarding Attitude toward online advertising, adblocker disablers scored significantly higher than filter bypassers who, in turn, scored higher than website leavers, all of which support H1. The magnitude of the difference between adblocker disablers and website leavers was large because its $\operatorname{Exp}(B)$ indicated a $101 \%$ increase in the odds ratio each time the predictor increased by one unit. Concerning Adblock usage experience, adblocker disablers scored lower than filter bypassers who, in turn, scored higher than website leavers, all of which satisfy H2. But the first difference had a medium magnitude (17\% reduction in odds) and the second a moderate magnitude ( $6 \%$ increase in odds). Moreover, adblocker disablers unexpectedly scored lower than website leavers with a moderate magnitude (12\% reduction in odds). In relation to Breadth of online activities, filter bypassers scored higher than the other groups and website leavers scored lower than the other groups, as predicted by H3, but these differences had small magnitudes. With respect to Online privacy concern, adblocker disablers scored lower than both filter bypassers and website leavers, as suggested by H4, with moderate magnitudes.

\section{Discussion}

The combination of psychological reactance theory along with uses and gratifications theory provided an appropriate conceptual framework to understand responses to anti-adblock filters. Whenever access to websites was restricted by anti-adblock filters, the affected users became motivated both to maintain control over online advertising and to achieve the content gratifications sought. In the formation of their response, these users considered the advantages and disadvantages of each option according to their knowledge, skills, experiences, beliefs, and habits.

Bypassing the ad-block filter was the most advantageous response for users because it allowed for finding both the content gratification (access to content) and the process gratification (control of advertising), which meant a complete correspondence between what was sought and what was obtained. However, this response required levels of knowledge and skills that only a minority of adblock users could afford (in fact, only 15\% of participants reported trying to bypass ad-block filters).

Disabling the adblocker implied gaining the content gratification but losing the process gratification, which created a partial mismatch between desired and actual outcomes. This mismatch put subjects in a less stable situation than that produced by the previous response. Therefore, although the prevalence of this response was quite high (44\%), a part of adblocker disablers are expected to become filter bypassers as they gradually accumulate sufficient knowledge and skills.

Leaving the website implied losing the desired content and process gratifications, an unstable situation in which the total mismatch with desires could conceivably predispose users to more advantageous responses. Thus, the fairly high prevalence of website leavers (40\%) is expected to be reduced as they find the conditions to bypass the anti-adblock filter (competence acquisition) or disable the adblocker (advertising acceptance). 
User choice among these responses was hypothesized to be driven by two attitudinal and two behavioral factors. Empirical support was found for all the hypothesized relationships, but the observed effect sizes were not always strong enough.

Attitude toward online advertising was by far the most influential factor in the responses to anti-adblock filters. This factor is particularly important in explaining the decision between disabling the adblocker and leaving the website. On the one hand, adblock users with more favorable attitudes toward online advertising tended to accept advertising exposure more easily and to feel less threatened by the loss of control over advertising, all of which facilitated their decision to disable the adblocker in order to access the website's content with its corresponding ads. On the other hand, adblock users with more unfavorable attitudes toward online advertising tended to experience a greater psychological reactance in the face of a possible loss of control over advertising and, as their desire not to receive advertising could even overshadow their desire to access the website's content, they tended to leave the website more easily.

Adblock usage experience was the second most influential factor in the responses to anti-adblock filters. As suggested by H2, filter bypassers scored higher on this factor than the other groups because individuals with longer adblock usage experience were able to accumulate the perceived and actual abilities that are typically required to bypass anti-adblock filters. However, website leavers scored higher than adblocker disablers, which is somewhat counterintuitive because the website abandonment does not require any skills, while the adblocker deactivation is more likely when users are familiar with adblocker operation (e.g., functional and temporal scope of each deactivation and ability to reverse each deactivation). A tentative explanation for this unexpected finding is that netizens with greater adblock usage experience were more inclined to leave the website because they had accumulated more skills that allowed them to find the same or similar content on alternative websites without anti-adblock filters.

Breadth of online activities was the least influential factor, though two expected effects were found. Firstly, netizens accustomed to satisfying a greater variety of content and process gratifications tended to be both more ambitious and more qualified to address the challenge of bypassing the anti-adblock filter with the double purpose of accessing the website's content and maintaining their control over advertising. Secondly, netizens accustomed to satisfying a smaller variety of gratifications tended to have less ability to address complicated challenges on the Internet, and so they were more inclined simply to leave the website, though this response implied not satisfying the content and process gratifications sought in their web browsing. Nevertheless, as the size of both effects was small, the influence of this factor can be considered negligible in practice.

Online privacy concern had a moderate negative effect on adblocker deactivation. Understandably, this concern aroused psychological reactance toward online advertising when netizens were aware that their browsing might be tracked by third-party advertising companies and feared that personal information collected in such a way might be used without their consent and control. Netizens feeling threatened by this loss of privacy control tended to protect themselves by responding with a complete rejection of the advertising, that is, by preventing the adblocker deactivation. 
The managerial implications for online advertising stakeholders are discussed in the following.

For ad-supported websites, what are the effects of using or not using anti-adblock filters? On the one hand, websites not using anti-adblock filters will lose the monetizable ad impressions from adblock users (who comprise $54 \%$ of the sample) but maintain the totality of website visitors and the possibility of triggering impressions to these through other advertising formats, such as sponsored content. On the other hand, websites using anti-adblock filters will recover the monetizable ad impressions from adblocker disablers (23\%) but lose the visits of website leavers (21\%), to whom no advertising or non-promotional content can be shown.

Since both alternatives produce positive and negative effects, what decision is recommended for ad-supported websites? It is advisable to use primarily an economic criterion, by which the alternative that provides more income for the set of advertising formats used is chosen. But non-economic criteria also have their place and could even be prioritized depending on situations. For example, some ad-supported websites could ignore anti-adblock filters, even if this implies a loss of advertising revenues, in order not to lose visitors and thus not reduce their desired social impact.

Websites that choose to use anti-adblock filters, and other involved stakeholders such as advertisers and ad agencies, are recommended to address unfavorable attitudes toward online advertising and concerns about online privacy, two factors identified in this study as detrimental to the desired performance of anti-adblock filters.

Improving attitudes toward online advertising should be a priority because this stands out as the most influential predictor of the differences between website leavers and adblocker disablers. An increase in the average valuation of online advertising would greatly stimulate both an increase in adblocker deactivation and a reduction in website abandonment. If involved stakeholders manage to avoid the worst-rated aspects of advertising (intrusiveness, annoyance, clutter, etc.) and the worst-rated formats (pop-up ads, auto-playing video ads with sound, non-skippable video ads, etc.), adblock users will experience lower levels of psychological reactance to anti-adblock filters and thus will be less tempted to reject advertising in a radical way (leaving the website) and more prone to accept being exposed to the website's ads (disabling the adblocker). In turn, if stakeholders manage to encourage the best-rated aspects of advertising (entertainment, information, creativity, etc.), advertising avoidance will be less perceived as a kind of process gratification, and so ads might even be intentionally searched for as content that provides the gratifications desired by netizens. But, nonetheless, improving attitudes toward online advertising is a difficult challenge because the malpractice of a few stakeholders can easily overshadow the good work of the majority and ultimately continue to feed user prejudices.

Addressing online privacy concerns should receive less attention because this factor only has a moderate influence on adblocker deactivation decrease. In order to reduce these concerns, stakeholders should avoid disrespectful practices (non-consensual data collection, misuse of personal data, unauthorized secondary use, etc.) and let netizens know. However, regaining netizen confidence may become a more difficult task after recent changes in the environment. A few years ago, recommendations were made to reduce online privacy concerns by empowering netizens to have more knowledge and control over their personal in- 
formation through website notifications of data collection practices and requests of informed consent (Aguirre et al., 2015; Boerman et al., 2017). The perception of this empowerment made netizens become less distrustful and ultimately more likely to be persuaded by website ads (Tucker, 2014). Since May 2018, the General Data Protection Regulation has greatly empowered European Union citizens by making their informed consent mandatory when their personal data is collected/used/transferred as well as by recognizing the right to revoke their consent at any time (EUR-Lex, 2016). This legal-based empowerment could probably change EU netizens' perceptions of this issue: respectful practices in the collection/use/transfer of personal information could begin to be considered as mere legal obligations of advertising stakeholders, while disrespectful practices, previously deemed unethical, could begin to be regarded as illegal.

In this complex environment, online advertising stakeholders face two additional difficulties. Firstly, adblocker producers are constantly improving their products with more sophisticated tools that flexibly adapt to the preferences of netizens, who then are empowered to choose (a) between allowing and blocking the ads meeting acceptability criteria and (b) between allowing and disabling third-party web tracking practices (Mazel et al., 2019). So, the use of adblockers can be expected to continue to spread in the coming years. Secondly, as the results suggest, the amount of filter bypassers (8\%) will increase as adblock users gain more usage experience. Moreover, the rapid race to develop more effective anti-adblock filter killers (Zhu et al., 2019) could encourage more and more adblock users to become filter bypassers. So, advertising stakeholders face a growing group that will not generate monetizable views of conventional online ads but will continue to view other advertising and non-promotional content shown in the website.

All things considered, it is advisable that ad-supported websites not only focus on trying to avoid the loss of conventional ad impressions but also on seeking alternative sources of funding through less conventional advertising formats such as sponsored content and "native ads" (i.e., promotional messages designed to look like non-promotional content), which generate monetizable impressions to all website visitors. In a somewhat similar way, advertisers who faced the progressive loss of TV viewers during commercial breaks chose to embed more and more promotional stimuli within non-promotional content, a practice that managed to lead to both the effectiveness of such stimuli and the support of content production (Redondo \& Bernal, 2016). Note that, consistent with the above discussion, less conventional formats should provide a rich browsing experience (through engaging information/entertainment content) and prevent inducing psychological reactance (by avoiding advertising clutter and other annoying practices).

\section{Conclusions}

Adblockers allow users to obtain both content gratifications (from the content of visited websites) and process gratifications (by avoiding the ads of such websites). Adblock users may experience psychological reactance whenever anti-adblock filters threaten their privileged position. In turn, anti-adblock filters allow websites to reduce their losses of ad impressions but simultaneously make websites lose a part of their visitors. These conflicting results are 
explained by the responses given by users to anti-adblock filters: adblocker disablers enable websites to recover monetizable ad impressions; website leavers produce losses of website visitors; and filter bypassers don't generate conventional ad impressions but their visits are monetizable through alternative advertising formats.

This study theoretically argued and empirically found that four user-related factors shape responses to anti-adblock filters. First, attitude toward online advertising plays a very important role because users favorably (unfavorably) predisposed to online advertising are considerably more inclined to accept (reject) ad impressions and thus opt for the adblocker deactivation (website abandonment). Second, adblock usage experience is a remarkable predictor because a longer experience enables users to acquire the knowledge and skills that are required to perform the complex task of bypassing anti-adblock filters. Third, breadth of online activities has a significant but negligible influence, by which users with a greater (lesser) breadth show levels of competence and motivation that support their willingness to perform the complex (simple) task of bypassing the anti-adblock filter (leaving the website). Fourth, online privacy concern is a moderate deterrent to adblocker deactivation because fear of misuse of personal data leads some netizens to reject ad impressions with which their browsing behavior could be tracked. Importantly, research on responses to anti-adblock filters is still very much in its infancy, and the sustainability of ad-supported websites calls for a much more extensive investigation of the influence of user attitudes/behaviors and website operations/strategies.

Regarding the limitations of this study, the participants were asked about their most frequent responses to anti-adblock filters, information that faithfully represented their typical behavior but completely ignored any possible variant of such behavior. Among other examples, those who usually left websites could have occasionally disabled the adblocker to access some website with exclusive or very attractive content; those who habitually disabled their adblockers could have abandoned some website where they had had previous negative experiences with advertising; and those who typically tried to bypass anti-adblock filters could have disabled their adblockers to access some website with well-valued advertising formats.

Another limitation is the non-random, self-reported, and cross-sectional nature of the data collected in this study. Firstly, the non-random recruitment may have led to a sample of participants with an above-average use of and involvement in online activities. The use of random sampling in future studies will provide much greater levels of generalizability to their results. Secondly, the self-reported data may contain inaccuracies/mistakes that the respondents have reported intentionally/inadvertently. This limitation will be obviously overcome when the data is collected through direct observation. Thirdly, the cross-sectional data only provides a simplified static representation of the phenomenon under consideration. Future longitudinal studies could dynamically measure how adblock users change their responses (especially how the group of filter bypassers grows) and modify their whitelisted websites (especially those disabled in response to anti-adblock filters).

Furthermore, it would be worthwhile to examine potential behavioral differences across desktop computers, tablets, and smartphones, as well as potential cultural differences, such as the possibility of online privacy concern of US netizens being less than that of their EU counterparts and thus having a smaller impact on adblocker deactivation. But it is more im- 
portant and urgent to conduct experiments to gain a general understanding of how responses to anti-adblock filters are conditioned by subjective factors of netizens (degree of involvement in online activity, positive/negative proximate/remote experiences of website ads, etc.) and by objective features of stimuli (types of warning messages of anti-adblock filters, types of advertising formats of websites, etc.).

\section{Acknowledgements}

The authors wish to thank AIMC (Asociación para la Investigación de Medios de Comunicación) for its crucial support in managing the collection, monitoring, and processing of the data used in this study.

\section{Funding}

This research did not receive any specific grant from funding agencies in the public, commercial, or not-for-profit sectors.

\section{Author contributions}

Both authors contributed equally to the preparation of this manuscript.

\section{Disclosure statement}

The authors declare no conflict of interest.

\section{References}

Aguirre, E., Mahr, D., Grewal, D., de Ruyter, K., \& Wetzels, M. (2015). Unraveling the personalization paradox: The effect of information collection and trust-building strategies on online advertisement effectiveness. Journal of Retailing, 91(1), 34-49. https://doi.org/10.1016/j.jretai.2014.09.005

Aseri, M., Dawande, M., Janakiraman, G., \& Mookerjee, V. S. (2020). Ad-blockers: A blessing or a curse? Information Systems Research, 31(2), 627-646. https://doi.org/10.1287/isre.2019.0906

Bandura, A. (1978). Self-efficacy: Toward a unifying theory of behavioral change. Advances in Behavior Research and Therapy, 1(4), 139-161. https://doi.org/10.1016/0146-6402(78)90002-4

Bleier, A., \& Eisenbeiss, M. (2015). The importance of trust for personalized online advertising. Journal of Retailing, 91(3), 390-409. https://doi.org/10.1016/j.jretai.2015.04.001

Boerman, S. C., Kruikemeier, S., \& Borgesius, F. J. Z. (2017). Online behavioral advertising: A literature review and research agenda. Journal of Advertising, 46(3), 363-376. https://doi.org/10.1080/00913367.2017.1339368

Brehm, S. S., \& Brehm, J. W. (1981). Psychological reactance: A theory of freedom and control. Academic Press.

Compeau, D., Higgins, C. A., \& Huff, S. (1999). Social cognitive theory and individual reactions to computing technology: A longitudinal study. MIS Quarterly, 23(2), 145-158.

https://doi.org/10.2307/249749 
Dinev, T., \& Hart, P. (2004). Internet privacy concerns and their antecedents - measurement validity and a regression model. Behavior \& Information Technology, 23(6), 413-422. https://doi.org/10.1080/01449290410001715723

Eastin, M. S., \& LaRose, R. (2000). Internet self-efficacy and the psychology of the digital divide. Journal of Computer-Mediated Communication, 6(1), JCMC611. https://doi.org/10.1111/j.1083-6101.2000.tb00110.x

Edwards, S. M., Li, H., \& Lee, J.-H. (2002). Forced exposure and psychological reactance: Antecedents and consequences of the perceived intrusiveness of pop-up ads. Journal of Advertising, 31(3), 83-95. https://doi.org/10.1080/00913367.2002.10673678

EUR-Lex. (2016). Regulation (EU) 2016/679 of the European Parliament and of the Council of 27 April 2016 on the protection of natural persons with regard to the processing of personal data and on the free movement of such data, and repealing Directive 95/46/EC. https://eur-lex.europa.eu/eli/reg/2016/679/oj

Gordon, B. R., Jerath, K., Katona, Z., Narayanan, S., Shin, J., \& Wilbur, K. C. (2020). Inefficiencies in digital advertising markets. Journal of Marketing. https://doi.org/10.1177/0022242920913236

Hair, J. F., Black, W. C., Babin, B. J., \& Anderson, R. E. (2010). Multivariate data analysis: A global perspective $\left(7^{\text {th }}\right.$ ed.). Pearson Education.

Ham, C.-D. (2017). Exploring how consumers cope with online behavioral advertising. International Journal of Advertising, 36(4), 632-658. https://doi.org/10.1080/02650487.2016.1239878

Hargittai, E., \& Hinnant, A. (2008). Digital inequality: Differences in young adults' use of the internet. Communication Research, 35(5), 602-621. https://doi.org/10.1177/0093650208321782

Helsper, E. J., \& Eynon, R. (2013). Distinct skill pathways to digital engagement. European Journal of Communication, 28(6), 696-713. https://doi.org/10.1177/0267323113499113

Ji, P., \& Fu, W. W. (2013). Love Internet, love online content: Predicting Internet affinity with information gratification and social gratifications. Internet Research, 23(4), 396-413. https://doi.org/10.1108/IntR-08-2012-0155

Lim, W. M., \& Ting, D. H. (2012). E-shopping: An analysis of the uses and gratifications theory. Modern Applied Science, 6(5), 48-63. https://doi.org/10.5539/mas.v6n5p48

MacKenzie, S. B., \& Lutz, R. J. (1989). An empirical examination of the structural antecedents of attitude toward the ad in an advertising pretesting context. Journal of Marketing, 53(2), 48-65. https://doi.org/10.2307/1251413

Mazel, J., Garnier, R., \& Fukuda, K. (2019). A comparison of web privacy protection techniques. Computer Communications, 144, 162-174. https://doi.org/10.1016/j.comcom.2019.04.005

Miyazaki, A. D. (2008). Online privacy and the disclosure of cookie use: Effects on consumer trust and anticipated patronage. Journal of Public Policy \& Marketing, 27(1), 19-33. https://doi.org/10.1509/jppm.27.1.19

Novak, T. P., Hoffman, D. L., \& Yung, Y.-F. (2000). Measuring the customer experience in online environments: A structural modeling approach. Marketing Science, 19(1), 22-42. https://doi.org/10.1287/mksc.19.1.22.15184

Palmgreen, P., Wenner, L. A., \& Rosengren, K. E. (1985). Uses and gratifications research: The past ten years. In K. Rosengren, L. Wenner, \& P. Palmgreen (Eds.), Media gratifications research: Current perspectives (pp. 11-37). Sage.

Payne, G. A., Severn, J. J. H., \& Dozier, D. M. (1988). Uses and gratifications motives as indicators of magazine readership. Journalism Quarterly, 65(4), 909-913. https://doi.org/10.1177/107769908806500411

Redondo, I., \& Aznar, G. (2018). To use or not to use ad blockers? The roles of knowledge of ad blockers and attitude toward online advertising. Telematics and Informatics, 35(6), 1607-1616.

https://doi.org/10.1016/j.tele.2018.04.008 
Redondo, I., \& Bernal, J. (2016). Product placement versus conventional advertising: The impact on brand choice of integrating promotional stimuli into movies. Journal of Promotion Management, 22(6), 773-791. https://doi.org/10.1080/10496491.2016.1214205

Redondo, I., \& Charron, J.-P. (2013). The payment dilemma in movie and music downloads: An explanation through cognitive dissonance theory. Computers in Human Behavior, 29(5), 2037-2046. https://doi.org/10.1016/j.chb.2013.04.015

Rojas-Méndez, J. I., \& Davies, G. (2005). Avoiding television advertising: Some explanations from time allocation theory. Journal of Advertising Research, 45(1), 34-48. https://doi.org/10.1017/S0021849905050154

Rubin, A. M. (1983). Television uses and gratifications: The interactions of viewing patterns and motivations. Journal of Broadcasting, 27(1), 37-51. https://doi.org/10.1080/08838158309386471

Ruggiero, T. E. (2000). Uses and gratifications theory in the $21^{\text {st }}$ century. Mass Communication and Society, 3(1), 3-37. https://doi.org/10.1207/S15327825MCS0301_02

Shiller, B., Waldfogel, J., \& Ryan, J. (2018). The effect of ad blocking on website traffic and quality. RAND Journal of Economics, 49(1), 43-63. https://doi.org/10.1111/1756-2171.12218

Smock, A. D., Ellison, N. B., Lampe, C., \& Wohn, D. Y. (2011). Facebook as a toolkit: A uses and gratification approach to unbundling feature use. Computers in Human Behavior, 27(6), 2322-2329. https://doi.org/10.1016/j.chb.2011.07.011

Söllner, J., \& Dost, F. (2019). Exploring the selective use of ad blockers and testing banner appeals to reduce ad blocking. Journal of Advertising, 48(3), 302-312. https://doi.org/10.1080/00913367.2019.1613699

Speck, P. S., \& Elliott, M. T. (1997). Predictors of advertising avoidance in print and broadcast media. Journal of Advertising, 26(3), 61-76. https://doi.org/10.1080/00913367.1997.10673529

Stafford, M. R., \& Stafford, T. F. (1996). Mechanical commercial avoidance: A uses and gratifications perspective. Journal of Current Issues \& Research in Advertising, 18(2), 27-38. https://doi.org/10.1080/10641734.1996.10505049

Tucker, C. E. (2014). Social networks, personalized advertising, and privacy controls. Journal of Marketing Research, 51(5), 546-562. https://doi.org/10.1509/jmr.10.0355

Tudoran, A. A. (2019). Why do Internet consumers block ads? New evidence from consumer opinion mining and sentiment analysis. Internet Research, 29(1), 144-166. https://doi.org/10.1108/IntR-06-2017-0221

Wang, Y., \& Sun, S. (2010). Assessing beliefs, attitudes, and behavioral responses toward online advertising in three countries. International Business Review, 19(4), 333-344. https://doi.org/10.1016/j.ibusrev.2010.01.004

Youn, S., \& Kim, S. (2019). Understanding ad avoidance on Facebook: Antecedents and outcomes of psychological reactance. Computers in Human Behavior, 98, 232-244. https://doi.org/10.1016/j.chb.2019.04.025

Zhu, S., Iqbal, U., Wang, Z., Qian, Z., Shafiq, Z., \& Chen, W. (2019). ShadowBlock: A lightweight and stealthy adblocking browser. In Proceedings of the 2019 World Wide Web Conference (pp. 24832493). ACM Press. https://doi.org/10.1145/3308558.3313558 


\section{APPENDIX}

\section{Items used to measure the variables}

Attitude toward online advertising

- Advertising is necessary for the Internet to develop.

- Internet advertising is more interesting than other media advertising.

- I find Internet advertising more useful compared to other media advertising.

- I don't mind receiving Internet advertising if I can access free audiovisual content.

Online privacy concern

- I'm concerned about the use that can be made of the personal data I provide on the Internet.

- I worry about governments controlling what I do on the Internet.

- I worry about companies controlling what I do on the Internet.

- I don't install apps that request a lot of personal information.

Breadth of online activities

Reading of current news; watching online videos on sites like YouTube; checking of maps/ street guides; checking of weather forecasts; streaming online music; streaming movies/TV series; checking the listings of movies/shows; dealing with public administrations; searching for information on health issues; searching for financial information; watching delayed broadcasts of TV channels; filling out a survey; searching for courses, degrees, training, etc.; downloading of software; signing of online petitions; viewing of live TV networks on their websites/apps; listening to live radio stations on their websites/apps; visiting adult websites; downloading of movies/TV series/documentaries; searching for employment; commenting on politics, economy, etc.; playing online games; downloading of music; checking of traffic information; making videocalls/videoconferences; listening to on-demand radio podcasts; downloading of e-books; searching for housing to buy, rent, etc.; receiving information/news via RSS; betting on sports, casinos, lotteries, etc.; remote control of other computer resources; getting discount coupons; full access to newspapers/magazines; and searching for a romantic partner/dating. 